\title{
Confinement of Excitons for the Lowest Optical Transition Energies of Single Wall Carbon Nanotubes*
}

\author{
Ahmad R. T. Nugraha, ${ }^{\dagger}$ Kentaro Sato, and Riichiro Saito \\ Department of Physics, Tohoku University, Sendai 980-8578, Japan \\ (Received 4 July 2010; Accepted 22 October 2010; Published 6 November 2010)
}

\begin{abstract}
The confinement of excitons in a single wall carbon nanotube (SWNT) is discussed based on an analysis of the environmental effect on the optical transition energies, $E_{i i}$, measured by photoluminescence spectroscopy. We use the effective dielectric constant $\kappa$ as a function of nanotube diameter and exciton size that has also been used to reproduce excitonic transition energies $E_{i i}$ from resonance Raman spectroscopy experiments. When we focus our attention to the lowest transitions, $E_{11}$ region, we find a systematic deviation of the $\kappa$ values for semiconducting type-I and type-II carbon nanotubes. We suggest that the $E_{11}$ energies observed by photoluminescence are upshifted to the calculated $E_{11}$ energies due to the confinement of excitons in the SWNTs. Considering this effect, the same energy shift formula for the environmental effect as that for the the Raman spectroscopy results can be used to reproduce experimental $E_{i i}$ values from photoluminescence spectroscopy within a good accuracy, hence providing an accurate assignment of many nanotube chiralities. [DOI: 10.1380/ejssnt.2010.367]
\end{abstract}

Keywords: Many body and quasi-particle theories; Light scattering; Carbon nanotubes

\section{INTRODUCTION}

The optical transition energies $E_{i i}$, where $i=1,2,3, \ldots$ denotes the transition between the $i$-th valence and $i$-th conduction subbands measured from the Fermi level, and the radial breathing mode frequencies $\left(\omega_{\mathrm{RBM}}\right)$ of single wall carbon nanotubes (SWNTs) have been frequently used to assign their structural $(n, m)$ indices [1] based on photoluminescence (PL) and resonance Raman spectroscopy (RRS) measurements $[2,3]$. Knowing the $(n, m)$ index of a SWNT allows us to understand the physical properties of the SWNT which are strongly $(n, m)$ dependent. Thus it is of great importance to have an accurate description of $E_{i i}$ and $\omega_{\mathrm{RBM}}$ as a function of $(n, m)$. The concept of excitons has been widely accepted to explain the $E_{i i}$ energies, both from experimental and theoretical points of view, because the exciton binding energies of SWNTs are very large, up to $1 \mathrm{eV}$, so that the excitons survive even at the room temperature [4-8]. However, the excitonic transition energy $E_{i i}$ for a given SWNT is known to be strongly affected by a change in the surrounding materials around the SWNT, which is called the environmental effect [9].

While the environment effect on $\omega_{\mathrm{RBM}}$ 's has been understood by some empirical formulas [10, 11], a complete description of the $E_{i i}$ 's environmental effect is still under development, though we now almost get the final conclusion for it [12], where one has to consider the excitonic dielectric screening effect of $E_{i i}$ by the surrounding materials. The "Kataura" plot $[13,14]$, in which $E_{i i}$ as a function of the nanotube diameter $d_{t}$, will be different for one particular environment relative to another, so a general picture for explaining the environmental effect is needed. In the previous works $[11,12]$, the dielectric constant $\kappa$ is theoretically expressed as a function of $d_{t}$ and also exciton size $l_{k}$ in reciprocal space that can reproduce

*This paper was presented at SSSJ-A3 Foresight Joint Symposium on Nanomaterials and Nanostructures, Hongo Campus, The University of Tokyo, Tokyo, Japan, 7-9 July, 2010.

†Corresponding author: nugraha@flex.phys.tohoku.ac.jp many $E_{i i}$ values observed by RRS measurements, namely, $E_{11}^{\mathrm{S}}, E_{22}^{\mathrm{S}}, E_{11}^{\mathrm{M}}, E_{33}^{\mathrm{S}}$ and $E_{44}^{\mathrm{S}}$, where $\mathrm{S}$ and $\mathrm{M}$ denote semiconducting and metallic SWNTs, respectively. Not only was the method successfully applied to many $E_{i i}$ values of many $(n, m)$ SWNTs synthesized by a particular environmental conditon, but it has also been proven to work well for different nanotube environments. In this study, we apply the same treatment to some PL $E_{i i}$ data $[15,16]$, and we find it is applicable, too, for these datasets. However, we notice that there is a deviated tendency for the $\kappa$ values for type-I $(\bmod (2 n+m, 3)=1)$ and type-II $(\bmod (2 n+m, 3)=2)$ semiconducting SWNTs for $E_{11}^{\mathrm{S}}$ only in the PL case. This deviation was not recognized previously in the RRS case because of a limited number of $E_{11}^{\mathrm{S}}$ data. Here we suggest a model based on the confinement effect of excitons in a SWNT.

\section{THEORETICAL METHOD}

The details of the exciton energy calculation used here have been described in some previous papers [17-19]. $E_{i i}$ is calculated as a transition from the ground state to the first bright exciton state by solving the Bethe-Salpeter equation within the extended tight-binding (ETB) model. The ETB model includes the effect of curvature (single particle energy $E_{\mathrm{sp}}$ ) and many-body effects (self energy $\Sigma$ and exciton binding energy $E_{\mathrm{bd}}$ ), so that the exciton energy can be calculated as $E_{\mathrm{ex}}=E_{\mathrm{sp}}+\Sigma-E_{\mathrm{bd}}$. The Coulomb interaction is essential for considering the environmental effect since the shift of $E_{i i}$ is understood by the change of $\kappa$ in which the unscreneed Coulomb potential $V$ between carbon $\pi$ electrons is modeled by the Ohno potential $[4,5,22]$, while the static screened Coulomb potential is expressed as $W=V / \kappa \epsilon(\vec{q})$, where $\epsilon(\vec{q})$ is the polarization function for the $\pi$ electrons. The effect of electrons in core states, $\sigma$ bonds, and the surrounding materials are all represented by a single parameter $\kappa$. Thus, the expression for $W$ can be understood as the origin of the variation of $E_{i i}$ due to a change to the surrounding materials, because $W$ is a main constituent in the $\Sigma$ and $E_{\text {bd }}$ calculations [17].

Previously, a constant $\kappa=2.22$ was used by Jiang et al. 
to describe $E_{i i}$ of bundled SWNTs for a limited range of diameter, $1.0<d_{t}<1.6 \mathrm{~nm}[17,18]$. Miyauchi et al. then used $1 / \kappa=C_{\text {tube }} / \kappa_{\text {tube }}+C_{\text {env }} / \kappa_{\text {env }}$, where $C_{\text {tube }}$ and $C_{\text {kappa }}$ are $d_{t}$-dependent coefficients, and succesfully reproduced some experimental $E_{i i}$ values, though only for a very few number of PL $E_{11}^{\mathrm{S}}$ energies [20]. Araujo et al. recently reported a $d_{t}$-dependent $\kappa$ that could reproduce many experimental $E_{i i}$ values and thus represents a breakthrough toward tackling the environmental effect [21]. However, different $\kappa$ dependencies on $d_{t}$ were obtained for $\left(E_{11}^{\mathrm{S}}, E_{22}^{\mathrm{S}}, E_{11}^{\mathrm{M}}\right)$ relative to $\left(E_{33}^{\mathrm{S}}, E_{44}^{\mathrm{S}}\right)$. Then, we have improved the $\kappa$ function to include its dependence on the exciton size $l_{k}$ in reciprocal space [12]. The adopted model for the $\kappa$ function is

$$
\kappa \approx C_{\kappa}\left[p^{0.8}\left(1 / d_{t}\right)^{1.6}\left(1 / l_{k}\right)^{0.4}\right],
$$

where the integer $p$ corresponds to the ratio of the distances of the cutting lines for each $E_{i i}$ transition from the $\mathrm{K}$ point in 2D Brillouin zone of graphene [14], and $p=1,2,3,4,5$ stand for $E_{11}^{\mathrm{S}}, E_{22}^{\mathrm{S}}, E_{11}^{\mathrm{M}}, E_{33}^{\mathrm{S}}$, and $E_{44}^{\mathrm{S}}$, respectively. The variable $l_{k}$ is involved in the $\kappa$ function because of the screening by the different environments (and metallicity of SWNTs) which modifies the exciton size. The fitted powers $(0.80 \pm 0.10,1.60 \pm 0.10,0.40 \pm 0.05)$ for $\left(p, 1 / d_{t}, 1 / l_{k}\right)$ have been determined so as to both optimize the correlation between $\kappa$ and $\left(p, d_{t}, l_{k}\right)$, and to minimize $E_{i i}$ differences between theory and experiment [12]. The powers also indicate another scaling relation of excitons similar to the previously reported scaling law which relates the exciton binding energy with the nanotube diameter, dielectric constant, and effective mass [22]. However, the use of the effective mass does not work to describe the difference between M- and S-SWNTs because the polarization functions $\epsilon(\vec{q})$ for M- and S-SWNTs are significantly different from each other even for a similar $m^{*}$.

The parameter $C_{\kappa}$ is expressed directly by the gradient or slope of the $\kappa$ function with an assumption that each $C_{\kappa}$ characterizes the environmental dielectric constant $\kappa_{\text {env }}$ of each sample, such as determined by different nanotube synthesis methods or particular solvents and wrapping materials around the tubes [12]. Previously we adopted the experimental $E_{i i}$ values of resonance Raman excitation profile for super-growth (SG) [21, 23], alcoholcatalytic CVD (ACCVD) [21, 24], and HiPco [9] based SWNTs. We have set the SG sample as a standard because it has the largest $E_{i i}$ and hence the smallest $\kappa$ compared to the other samples [23], so that the $C_{\kappa}$ values for all other samples should be normalized to the SG's $C_{\kappa}$, giving $\tilde{C}_{\kappa}=C_{\kappa} / C_{\kappa}^{\mathrm{SG}}$ (thus $\tilde{C}_{\kappa}^{\mathrm{SG}}=1$ ). Now for the PL treatment we use $E_{i i}$ data from the work by Weisman et al. [15] and Ohno et al. [16] which give SWNTs under three different environments: (i) HiPco SWNTs dispersed in sodium decodyl sulfate (SDS) aquaeous solution [15], (ii) ACCVD trench-suspended SWNTs immersed in hexane (HEX) [16], and (iii) ACCVD trenchsuspended SWNTs immersed in chloroform (CL) [16].

\section{RESULTS AND DISCUSSION}

In PL spectra, $E_{11}$ energies are given by the emission spectra, while the other higher $E_{i i}$ energies $(i=$
$2,3,4, \ldots)$ are given by the absorption spectra. Since there are no PL spectra for M-SWNTs, only S-SWNTs are considered here. As were obtained previously for the RRS $E_{i i}$ data [12], in Fig. 1 it is now given a series of fitted $\kappa$ as a function of $\left(p, d_{t}, l_{k}\right)$ obtained from the PL $E_{i i}$ data. For each sample, all the $E_{i i}$ transitions are unified into a single linear $\kappa$ function with a slope $C_{\kappa}$ as indicated by the violet lines in Fig. 1(a)-(c). The normalized slope values $\tilde{C}_{\kappa}$ are also mentioned in the figure and they are compared to the RRS data as are given in Table I. In particular, since it is known that the $\kappa_{\text {env }}$ value for chloform is higher than that for hexane [16], the $\tilde{C}_{\kappa}$ values obtained for these two samples also follow the same behavior. This fact strengthens the previous assumption that $C_{\kappa}$ (or $\left.\tilde{C}_{\kappa}\right)$ characterizes the environmental dielectric constant of the samples. The physical assumption of $\tilde{C}_{\kappa}$ can also be justified by dividing $\kappa$ of each sample with its respective $\tilde{C}_{\kappa}$ as shown in Fig. $1(\mathrm{~d})$, in which all $\kappa$ data collapse on to a single line. Another point to note is that the $\tilde{C}_{\kappa}$ values for HiPco SDS samples measured by PL and RRS spectroscopy are similar to each other, indicating our $\kappa$ model can be safely used for both PL and RRS.

With the knowledge of $\tilde{C}_{\kappa}$ for several types of environments, we can use these results in practical applications. To a first approximation, we fit the energy shift $\delta E_{i i}^{\mathrm{env}}$ due to different environments by:

$$
\begin{aligned}
\delta E_{i i}^{\mathrm{env}} & =E_{i i}^{\mathrm{SG}}-E_{i i}^{\mathrm{env}} \\
& \equiv \tilde{C}_{\kappa}\left[A+B\left(\frac{p}{d_{t}}\right)+C\left(\frac{p}{d_{t}}\right)^{2}\right],
\end{aligned}
$$

where $A, B, C$, are parameters common to all types of environments and $E_{i i}^{\text {env }}$ is calculated using the $\kappa$ functions. In the previous work [12], Eq. (2) with its corresponding $A, B$, and $C$ were obtained by considering only the RRS data. The best fits for $A, B$, and $C$ were found to be $-42.80 \pm 1.26 \mathrm{meV}, 46.34 \pm 1.32 \mathrm{meV} \cdot \mathrm{nm}$, and $-7.47 \pm 0.65 \mathrm{meV} \cdot \mathrm{nm}^{2}$, respectively. Now we find this equation is also applicable for the $\mathrm{PL}$ data, with $A=-40.10 \pm 1.08 \mathrm{meV}, B=47.22 \pm 1.47 \mathrm{meV} \cdot \mathrm{nm}$, and $C=-6.87 \pm 0.36 \mathrm{meV} \cdot \mathrm{nm}^{2}$. Here the $E_{i i}$ values for the SG sample obtained in RRS measurements are used as a standard because of the SG's smallest environmental dielectric constant among the present data. All $E_{i i}$ values for the other environments are then given by $E_{i i}^{\mathrm{env}}=E_{i i}^{\mathrm{SG}}-\delta E_{i i}^{\mathrm{env}}$. This treatment thus provides a general way to obtain the Kataura plot for SWNTs in any type of environment. A good accuracy of $E_{i i}^{\exp }-E_{i i}^{\text {cal }}$ is obtained within $50 \mathrm{meV}$ for all energy regions (about $0.9-3.0 \mathrm{eV})$ and diameter $\left(0.7<d_{t}<2.5 \mathrm{~nm}\right)$, as shown in Fig. 2.

Unlike the RRS measurements that can give a set of $\left(E_{11}^{\mathrm{S}}, E_{22}^{\mathrm{S}}, E_{11}^{\mathrm{M}}, E_{33}^{\mathrm{S}}, E_{44}^{\mathrm{S}}\right)$, the PL data shown in Fig. 1 only give $E_{11}^{\mathrm{S}}$ and $E_{22}^{\mathrm{S}}$, but the number of $E_{11}^{\mathrm{S}}$ data observed in PL measurements are much more than those in RRS measurements. We can then analyze the $E_{11}^{\mathrm{S}}$ data more carefully. Especially, when we look at the $E_{11}^{\mathrm{S}}$ region, denoted by circles in Fig. 1, there is a deviated tendency of the $\kappa$ values for type-I (red circles) and type-II (blue circles) SWNTs. For $E_{11}^{\mathrm{S}}$, since the type-I SWNTs have larger effective mass than type-II SWNTs [14], we expect a smaller exciton size for type-I SWNTs in real space. This means that the type-I SWNTs must have smaller 

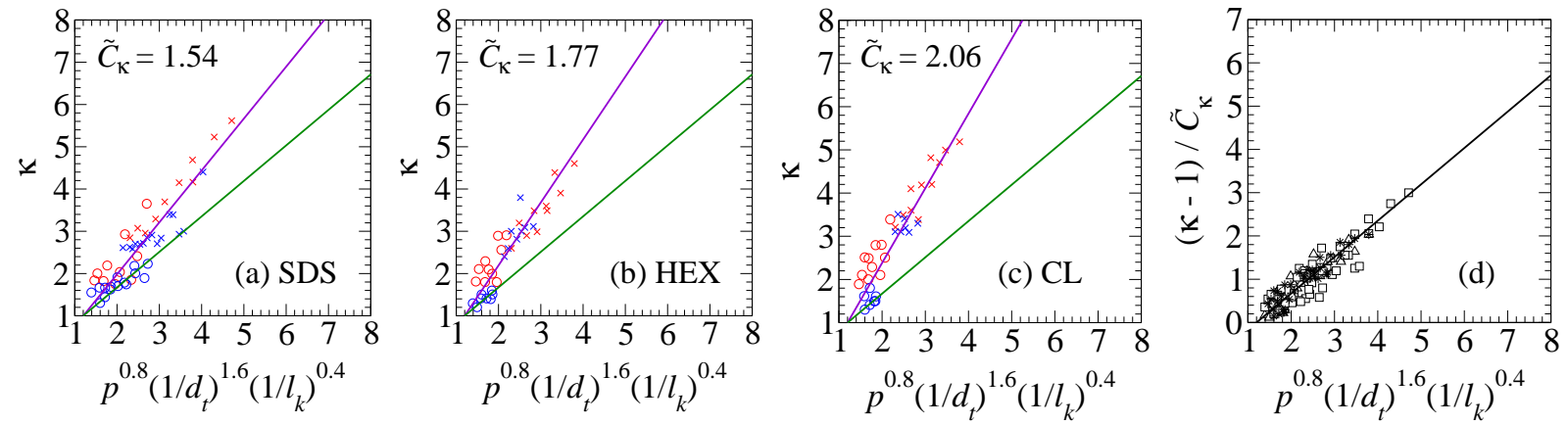

FIG. 1: The $\kappa$ function obtained from the PL $E_{i i}$ data. Panel (a) is for the HiPco SWNTs dispersed in SDS aqueous solution. Panels (b) and (c) are for the ACCVD trench-suspended SWNTs immersed in hexane and chloroform, respectively. Circle (cross) symbols are for $E_{11}\left(E_{22}\right)$. Red (blue) colors denote semiconducting type-I (type-II) SWNTs. A green line in each plot is a fitted line for the SG sample as a reference. This line is also a guide for eyes to distinguish the slope for different sample. The effective $\kappa$ values in (a) are determined from the $E_{i i}$ data in Ref. [15], whereas for those in (b) and (c) are determined from the $E_{i i}$ data in Ref. [16]. Panel (d) shows all $\kappa$ values from (a)-(c) divided by their corresponding $\tilde{C}_{\kappa}$. Squares, triangles, and stars are for SDS, HEX, and CL, respectively.

TABLE I: List of $\tilde{C}_{\kappa}$ values obtained within our $\kappa$ function method.

\begin{tabular}{ccccccc}
\hline \hline Measurement & \multicolumn{3}{c}{ RRS } \\
\hline $\begin{array}{c}\text { Synthesis method } \\
\text { (Environment) }\end{array}$ & $\begin{array}{c}\text { SG } \\
\text { (as-grown) }\end{array}$ & $\begin{array}{c}\text { ACCVD } \\
(\text { as-grown })\end{array}$ & $\begin{array}{c}\text { HiPco } \\
\text { (SDS) }\end{array}$ & $\begin{array}{c}\text { HiPco } \\
\text { (SDS) }\end{array}$ & $\begin{array}{c}\text { ACCVD } \\
\text { (HEX) }\end{array}$ & $\begin{array}{c}\text { ACCVD } \\
(\mathrm{CL})\end{array}$ \\
\hline$\tilde{C}_{\kappa}$ & $1.00 \pm 0.08$ & $1.42 \pm 0.03$ & $1.52 \pm 0.05$ & $1.54 \pm 0.05$ & $1.77 \pm 0.04$ & $2.06 \pm 0.06$ \\
\hline \hline
\end{tabular}

$\kappa$ values related to the previous explanation about the electric field lines created by excitons. In fact, if the exciton size is small, only small amount of the electric field can be affected by the environment. However, in Fig. 1, especially in panels (b) and (c), it is clear that type-I SWNTs tend to have larger $\kappa$ values compared to type-II SWNTs. This opposite behavior suggests that the exciton might be thermally activated by the center-of-mass motions in a finite SWNT length coupled with phonons, so that the $E_{11}^{\mathrm{S}}$ energies obtained from PL measurements are upshifted from the calculated $E_{11}^{\mathrm{S}}$ energies. The upshift value should be larger (smaller) for smaller (larger) effective mass, which will be shown as follows.

In the PL process, the excitons are relaxed from the $E_{22}^{\mathrm{S}}$ states to the $E_{11}^{\mathrm{S}}$ states by the exciton-phonon interaction. This interaction might not perfectly relax the excitons to the lowest exciton states before the electrons and

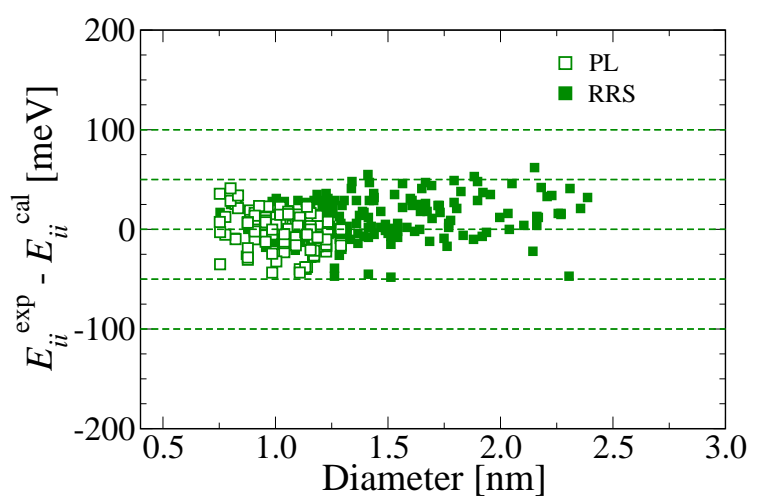

FIG. 2: Evaluation of the differences between experimental (exp) and calculated (cal) $E_{i i}$ values for all samples, showing good agreement between experiment and our model. Open and filled squares are for PL and RRS data, respectively. hole recombination gives the emitted light. The exciton state before the recombination is thus slightly upshifted in energy than the real lowest exciton state considered in the calculation because of the quantum confinement of an exciton. We denote this energy difference by $\Delta E_{11}^{\mathrm{S}}$ that can be understood as the energy upshift of the $E_{11}^{\mathrm{S}}$ in the plot of $\kappa$. Since $\kappa$ is obtained from the experimental $E_{11}^{\mathrm{S}}$ energies, the $\Delta E_{11}^{\mathrm{S}}$ values should shift the effective $\kappa$ depending on the tube type. A good parameter for this situation is the center-of-mass $M_{\mathrm{CM}}=\left(m_{\mathrm{e}}^{*}+m_{\mathrm{h}}^{*}\right) / 2$ (here $m_{\mathrm{e}}^{*}$ and $m_{\mathrm{h}}^{*}$ are the electron and hole effective masses, respectively), because $M_{\mathrm{CM}}$ of a type-I SWNT is generally larger than that of a type-II SWNT for a similar $d_{t}$. Then, we expect that $\Delta E_{11}^{\mathrm{S}} \sim \hbar^{2} k_{\mathrm{CM}}^{2} / 2 M_{\mathrm{CM}}$ for the type-I SWNTs should be smaller than that for the type-II SWNTs, so that the $\kappa$ values for type-I SWNTs will be upshifted smaller. This will result in the correct tendency of the $\kappa$ values, that is, the $\kappa$ values for type-I SWNTs are smaller than those for type-II SWNTs, or at least if we cannot make it, the $\kappa$ values for both type-I and type-II SWNTs are not separated too much.

We can make a model for $\Delta E_{11}^{\mathrm{S}}$ by considering the exciton motion is restricted in a finite length, analogous to the problem of a particle in a box. $\Delta E_{11}^{\mathrm{S}}$ is expressed as

$$
\Delta E_{11}^{\mathrm{S}}=\frac{\int_{0}^{\infty} E D(E) f(E) \mathrm{d} E}{\int_{0}^{\infty} D(E) f(E) \mathrm{d} E},
$$

where $D(E)$ is the electronic density of states and $f(E)=$ $\mathrm{e}^{-\left(E / k_{\mathrm{B}} T\right)}$ is the Boltzmann distribution function, $k_{\mathrm{B}}$ is the Boltzmann constant, and $T$ is temperature. If we approximate $D(E)$ with the $1 \mathrm{D}$ nanotube DOS, $D(E) \propto$ $1 / \sqrt{E}$, it will result in a constant $\Delta E_{11}^{\mathrm{S}}=\frac{1}{2} k_{\mathrm{B}} T$ at a given temperature, independent of $M_{\mathrm{CM}}$, which does not 


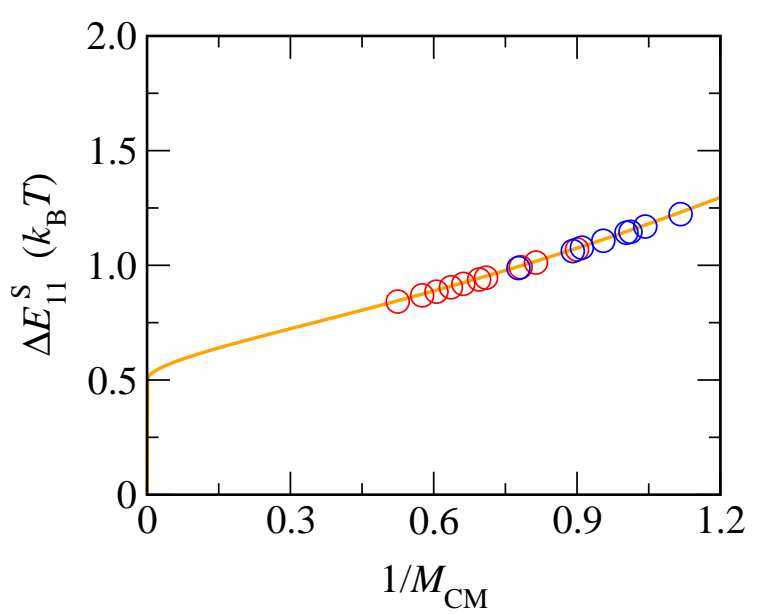

FIG. 3: $\Delta E_{11}^{\mathrm{S}}$ as a function of $M_{\mathrm{CM}}$ for the hexane PL data. To get the values shown in the horizontal axis, $1 / M_{\mathrm{CM}}$ should be multiplied by a factor $\left(\hbar^{2} \pi^{2} / 2 L^{2}\right) / k_{\mathrm{B}} T$. Red and blue circles denote type-I and type-II SWNTs, respectively. $L=20 \mathrm{~nm}$ and $T=300 \mathrm{~K}$ are used in this calculation.

explain the phenomena. Thus we have to model the exciton motion quantum mechanically as a particle in a box which gives discrete energy states $E_{n}$ as a function of center-of-mass:

$$
E_{n}=\frac{\hbar^{2} \pi^{2}}{2 M_{\mathrm{CM}} L^{2}} n^{2},
$$

where $L$ is length in which the exciton is confined. The DOS in Eq. (3) becomes a delta function and thus the integral turns to a summation:

$$
\Delta E_{11}^{\mathrm{S}}=\frac{\sum_{n=0}^{\infty} E_{n} \mathrm{e}^{-\left(E_{n} / k_{\mathrm{B}} T\right)} \mathrm{d} E}{\sum_{n=0}^{\infty} \mathrm{e}^{-\left(E_{n} / k_{\mathrm{B}} T\right)} \mathrm{d} E} .
$$

Since $E_{n}$ is inversely proportional to $M_{\mathrm{CM}}, \Delta E_{11}^{\mathrm{S}}$ is also roughly inversely proportional to $M_{\mathrm{CM}}$, as shown by a solid line in Fig. 3.

Therefore, we can achieve the expectation to have smaller (larger) $\Delta E_{11}^{\mathrm{S}}$ for the type-I (type-II) SWNTs because $M_{\mathrm{CM}}$ for the type-I (type-II) SWNTs is larger (smaller). A plot of $\Delta E_{11}^{\mathrm{S}}$ calculated from Eq. (3) for the hexane data is given in Fig. 3 with a fixed $L=20 \mathrm{~nm}$ and $T=300 \mathrm{~K}$. The $\Delta E_{11}^{\mathrm{S}}$ correction can then be applied to the determination of $\kappa$ from $E_{11}^{\mathrm{S}}$ original data,

$$
E_{11}^{\mathrm{S}}=E_{11}^{\mathrm{S}}(\exp )-\Delta E_{11}^{\mathrm{S}},
$$

where $E_{11}^{\mathrm{S}}$ is now the exciton state free of the center-ofmass motion, that can be used to determine the effective $\kappa$.

With the use of $L=20 \mathrm{~nm}, \Delta E_{11}^{\mathrm{S}}$ for type-I and typeII SWNTs are about $20-30 \mathrm{meV}$ and $25-35 \mathrm{meV}$, respectively. The corresponding $\kappa$ upshifts are then about $0.4-0.6$ and $0.5-0.7$ for type-I and type-II SWNTs, respectively. If we use larger $L$, for example $L=200 \mathrm{~nm}$ similar to a typical nanotube length found in experiments, $\Delta E_{11}^{\mathrm{S}}$ will be close to a constant $\frac{1}{2} k_{\mathrm{B}} T$. We conclude that the exciton motions are very restricted in a short finite length $L$ in the center part of the nanotube axis. We do not have a clear image why the exciton is confined in such a short region. A possible explanation is that the exciton is self-trapped by the lattice deformation or defects of a SWNT. These are open issues for the future work.

\section{CONCLUSION}

In conclusion, we have developed the $\kappa$ function for SWNTs surrounded by several different types of materials using a simple dielectric constant model. The treatment is applicable for $E_{i i}$ values obtained from both RRS and $\mathrm{PL}$ spectroscopy. For the $E_{11}^{\mathrm{S}}$ region in the PL case, the opposite behavior of the $\kappa$ values for type-I and type-II SWNTs is expected to originate from the confinement of exciton motions that makes the experimental $E_{11}^{\mathrm{S}}$ values are upshifted from the calculated values.

\section{Acknowledgments}

ARTN is supported by MEXT scholarship. RS acknowledges MEXT Grant (No. 20241023).
[1] R. Saito, G. Dresselhaus, and M. S. Dresselhaus, Physical Properties of Carbon Nanotubes (Imperial College Press, London, 1998).

[2] S. M. Bachilo, M. S. Strano, C. Kittrell, R. H. Hauge, R. E. Smalley, and R. B. Weisman, Science 298, 2361 (2002).

[3] A. Jorio, R. Saito, J. H. Hafner, C. M. Lieber, M. Hunter, T. McClure, G. Dresselhaus, and M. S. Dresselhaus, Phys. Rev. Lett. 86, 1118 (2001).

[4] T. Ando, J. Phys. Soc. Jpn. 66, 1066 (1997).

[5] C. D. Spataru, S. Ismail-Beigi, L. X. Benedict, and S. G. Louie, Phys. Rev. Lett. 92, 077402 (2004).

[6] F. Wang, G. Dukovic, L. E. Brus, and T. F. Heinz, Science 308, 838 (2005).

[7] J. Maultzsch, R. Pomraenke, S. Reich, E. Chang, D. Prezzi, A. Ruini, E. Molinari, M. S. Strano, C. Thom- sen, and C. Lienau, Phys. Rev. B 72, 241402 (2005).

[8] M. S. Dresselhaus, G. Dresselhaus, R. Saito, and A. Jorio, Annu. Rev. Phys. Chem. 58, 719 (2007).

[9] C. Fantini, A. Jorio, M. Souza, M. S. Strano, M. S. Dresselhaus, and M. A. Pimenta, Phys. Rev. Lett. 93, 147406 (2004).

[10] P. T. Araujo, C. Fantini, M. M. Lucchese, M. S. Dresselhaus, and A. Jorio, Appl. Phys. Lett. 95, 261902 (2009).

[11] P. T. Araujo, P. B. C. Pesce, M. S. Dresselhaus, K. Sato, R. Saito, and A. Jorio, Physica E 42, 1251 (2010).

[12] A. R. T. Nugraha, R. Saito, K. Sato, P. T. Araujo, A. Jorio, and M. S. Dresselhaus, Appl. Phys. Lett. 97, 091905 (2010).

[13] H. Kataura, Y. Kumazawa, Y. Maniwa, I. Umezu, S. Suzuki, Y. Ohtsuka, and Y. Achiba, Synthetic Metals 103, 2555 (1999). 
[14] R. Saito, G. Dresselhaus, and M. S. Dresselhaus, Phys. Rev. B 61, 2981 (2000).

[15] R. B. Weisman and S. M. Bachilo, Nano Lett. 3, 1235 (2003).

[16] Y. Ohno, S. Iwasaki, Y. Murakami, S. Kishimoto, S. Maruyama, and T. Mizutani, Phys. Status Solidi B 244, 4002 (2007).

[17] J. Jiang, R. Saito, Ge. G. Samsonidze, A. Jorio, S. G. Chou, G. Dresselhaus, and M. S. Dresselhaus, Phys. Rev. B 75, 035407 (2007).

[18] K. Sato, R. Saito, J. Jiang, G. Dresselhaus, and M. S. Dresselhaus, Phys. Rev. B 76, 195446 (2007).

[19] R. Saito, K. Sato, P. T. Araujo, A. Jorio, G. Dresselhaus, and M. S. Dresselhaus, Phys. Status Solidi B 246, 2581
(2009).

[20] Y. Miyauchi, R. Saito, K. Sato, Y. Ohno, S. Iwasaki, T. Mizutani, J. Jiang, and S. Maruyama, Chem. Phys. Lett. 442, 394 (2007).

[21] P. T. Araujo, A. Jorio, M. S. Dresselhaus, K. Sato, and R. Saito, Phys. Rev. Lett. 103, 146802 (2009).

[22] V. Perebeinos, J. Tersoff, and P. Avouris, Phys. Rev. Lett. 92, 257402 (2004).

[23] P. T. Araujo and A. Jorio, Phys. Status Solidi B 245, 2201 (2008).

[24] P. T. Araujo, S. K. Doorn, S. Kilina, S. Tretiak, E. Einarsson, S. Maruyama, H. Chacham, M. A. Pimenta, and A. Jorio, Phys. Rev. Lett. 98, 067401 (2007). 